\title{
Ultrastructural observations on the spermatozoa of a species of Lepidodasys (Gastrotricha, Macrodasyida)
}

\author{
L. Pierboni $\cdot$ R. M. Kristensen
}

Received: 22 September 2006 / Revised: 21 February 2007 / Accepted: 28 February 2007 / Published online: 12 April 2007

(C) Springer-Verlag and AWI 2007

\begin{abstract}
The ultrastructure of the spermatozoon of a species in the marine gastrotrich genus Lepidodasys is described. The filiform cell is composed of a cork-screw acrosome, a long single mitochondrion surrounded by a helical nucleus, and a flagellum with a $9 \times 2+2$ axonemal arrangement. The structure of the sperm of this species from Denmark appears closely similar to those of the other two species of Lepidodasys studied so far from Italy and Florida (US). Peculiar features (cylindrical nucleus, absence of a periaxonemal sheath) place this genus far from the others in the family Lepidodasyidae. The absence of synapomorphies between Lepidodasys and other genera of Lepidodasyidae suggests that the family is polyphyletic. The sperm ultrastructure fully fits the species of Lepidodasys into the marine order Macrodasyida, with the sperm ground plan of which its sperm shares a number of details.
\end{abstract}

Keywords Lepidodasys - Gastrotricha - Macrodasyida . Spermatozoa $\cdot$ Ultrastructure

Communicated by Peter Funch.

\section{Pierboni $(\square)$}

Istituto di Scienze Morfologiche,

Università di Urbino 'Carlo Bo',

Campus Scientifico, Località Crocicchia,

Via M. Oddi, 21, 61029 Urbino, Italy

e-mail: 1.pierboni@uniurb.it

R. M. Kristensen

Zoological Museum,

University of Copenhagen,

Universitetsparken 15 ,

2100 Copenhagen $\varnothing$, Denmark

\section{Introduction}

Gastrotrichs are aquatic, microscopic invertebrates, freeliving in periphytic, benthic and interstitial habitats. Of the about 700 known gastrotrich species, 240 belong to the marine order Macrodasyida, which includes 6 families and 30 genera. There are still uncertainties about the evolutionary relationships of these taxa, since literature data are still insufficient and often incomplete. In the last years some phylogenetic reconstructions, based on characters from both body anatomy and spermatozoon ultrastructure, as well as ones from 18S rRNA gene sequences, have outlined some possible scenarios of the intra-phyletic relationships, in particular within the order Macrodasyida (Hochberg and Litvaitis 2000, 2001; Todaro et al. 2003, 2006; Manylov et al. 2004; Marotta et al. 2005).

The family Lepidodasyidae (Macrodasyida) includes seven genera quite different in morphology and biology, and is considered as polyphyletic by Hochberg and Litvaitis (2001) and Marotta et al. (2005). In particular, Lepidodasys, despite it is the type genus of the family, differs from the other genera for a number of morphological characters: the complexity of the cuticle, the presence of Y-cells containing myofilaments, the non-striated pharyngeal myo-epithelium, the absence of circular musculature in the lateral body regions and the lack of pharyngeal pores (Rieger and Rieger 1977; Ruppert 1978). Even the ultrastructure of the mature spermatozoon differs in showing a ribbon-like nucleus, and in lacking any accessory structure around the axoneme (Guidi et al. 2004; Marotta et al. 2005).

Several recent studies have shown that gastrotrich spermatozoa are species-specific, and are useful phylogenetic characters (Balsamo et al. 1998; Balsamo and Todaro 2002; Guidi et al. 2003a, b, 2004; Marotta et al. 2005). Thus, the finding of a species of Lepidodasys different from those 
previously examined has prompted us to observe its spermatozoa for integrating the available data on the genus.

\section{Methods}

A single, adult specimen of an unidentified species of Lepidodasys was extracted from a sample of marine sediment collected in March 1976 in Kattegat at Ellekilde Hage, N$\mathrm{W}$ off Elsinore (Denmark) (Lat. 56 $05^{\prime} 83^{\prime \prime} \mathrm{N}$ and Long. $\left.12^{\circ} 30^{\prime} 01^{\prime \prime} \mathrm{E}\right)$. The sample was fixed in toto with a trialdehyde fixative mixture (Kalt and Tandler 1971), slightly modified for arthropod tissues (Lake 1973), and consisting of $2.9 \%$ glutaraldehyde, $2.0 \%$ formaldehyde, $1.25 \%$ acrolein and $2.6 \%$ dimethyl sulphoxide (DMSO) in $0.1 \mathrm{M}$ sodium cacodylate buffer at $\mathrm{pH}$ 7.4. The trialdehyde fixation was prepared according to the modified technique by Kristensen (1976) for tardigrades, which strongly adhere to the sediment. The whole sediment sample was bulk fixed in trialdehyde for $2 \mathrm{~h}$, thereafter the decanted supernatant was transferred to sucrose buffer with sodium cacodylate. The animal was sorted out in the sucrose buffer and thereafter postfixed in $1 \%$ osmium tetroxide (adjusted to $\mathrm{pH} 7.4$ with $0.1 \mathrm{M}$ sodium cacodylate buffer) for one hour at $20^{\circ} \mathrm{C}$. After fixation, the animal was dehydrated in an ethanol series, transferred to propylene oxide, and finally embedded in
Fig. 1 Ultrastructure of the spermatozoon of Lepidodasys sp. a Longitudinal section of the apical region (aa) of the acrosome. b Longitudinal section of the acrosome including the apical region and basal regions (ba). c Cross-section of the apical acrosomal region. d Longitudinal section of the basal region of the acrosome containing material organized into irregular vesicles (arrow). e Longitudinal section of the basal acrosomal region and of the nuclear base. $\mathbf{f}$ Cross-section of the basal region of the acrosome. $\mathbf{g}$ Longitudinal section of the nucleus-mitochondrion complex: the spiralized nucleus (n) surrounds the mitochondrion (arrows). h Longitudinal section of the 'connecting piece' (arrow head) lying in a cavity at the nuclear base. The mitochondrion is also visible (arrows). i Oblique and crosssection of the nucleus surrounding the mitochondrion (m). $\mathbf{j}$ Small electron-transparent spaces (s) with various size are regularly arranged into the condensed chromatin along the whole nuclear length. k Crosssection of the flagellum. I Schematic reconstruction of the mature spermatozoon
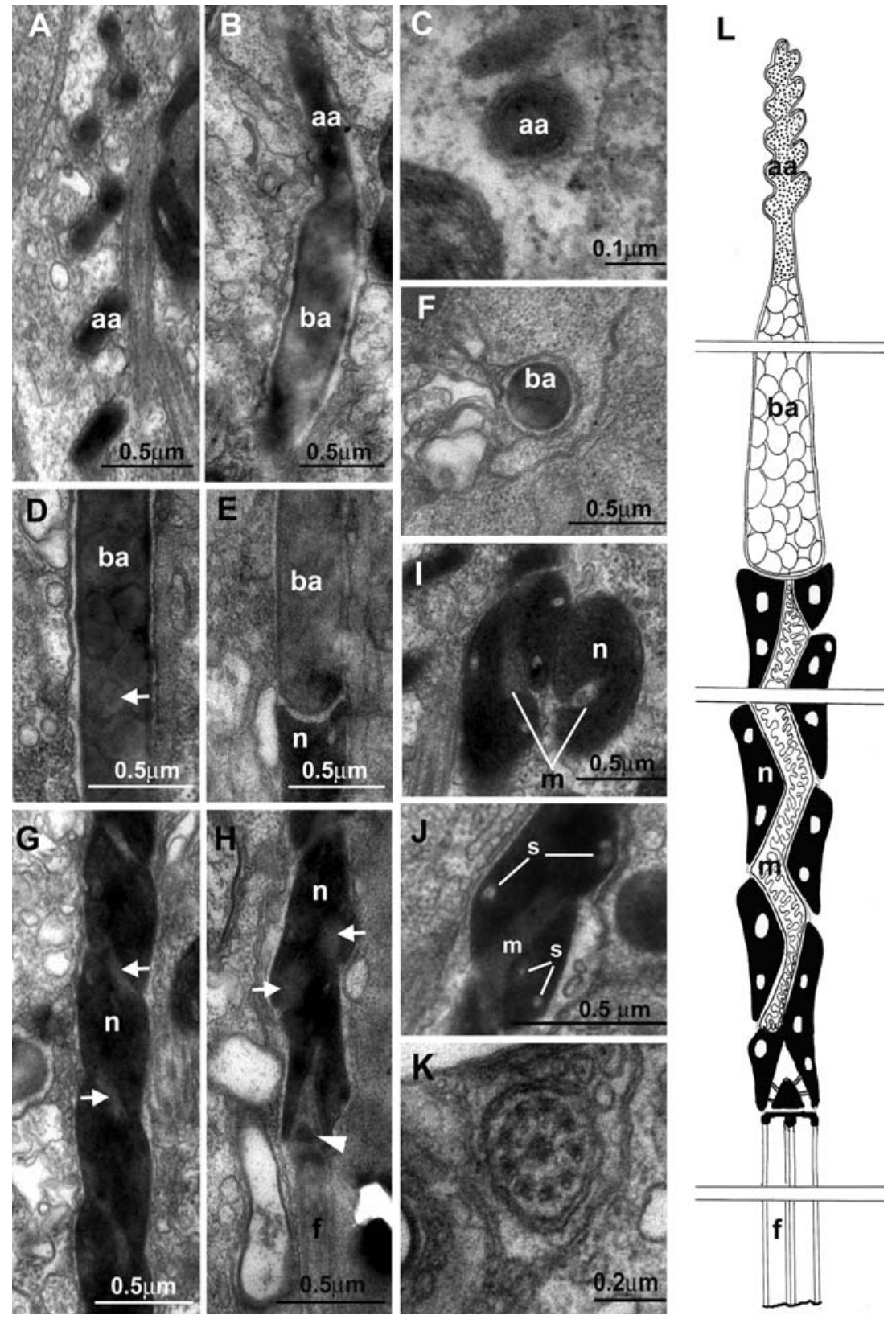
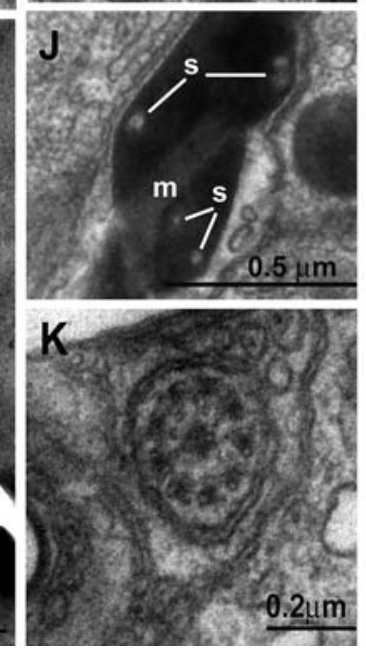

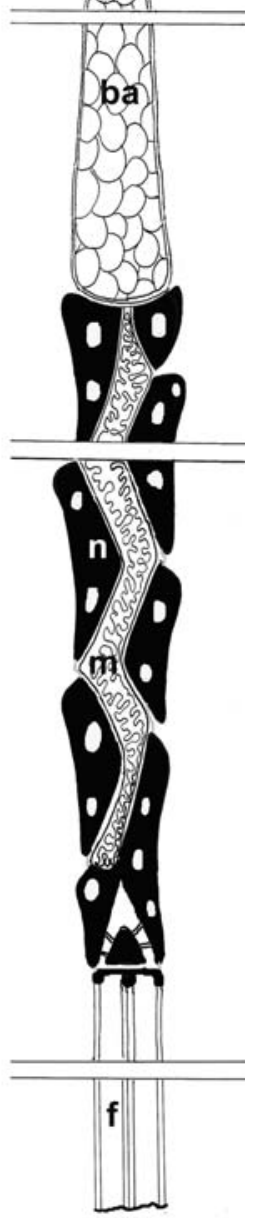


resin type Taab 812 (Epon 812). Ultrathin sections were cut with a diamond knife and stained with a saturated solution of $50 \%$ uranyl acetate in alcohol, followed by lead citrate solution (Reynold 1963). Sections were observed under a Jeol 100 SX transmission electron microscope.

\section{Results}

The mature spermatozoon of Lepidodasys sp. is a long and filiform cell, composed of an elongate acrosome, a nucleus surrounding a single mitochondrion, and a tail (Fig. 11). The acrosome is composed of two distinct regions. The apical one is cork-screw shaped, made up of 3-5 coils, and is at least $2 \mu \mathrm{m}$ in length and $0.14 \mu \mathrm{m}$ in diameter of (Fig. 1ac); it is filled with a very electron-dense material. The basal region has a rod-like shape, and measures about $3 \mu \mathrm{m}$ in length; its diameter, $0.34 \mu \mathrm{m}$, greatly decreases just at the transition to the apical region (Fig. 1b, d-f). It encloses numerous, large, and irregular vesicles with different diameters, tightly piled to each other containing a moderately electron-dense material. The ribbon-like nucleus, at least $10 \mu \mathrm{m}$ long, has a constant diameter, $0,5 \mu \mathrm{m}$, from the basal part to the apical one. It is a helix wounding a single, long, thin and twisted mitochondrion (Fig. $1 \mathrm{~g}-\mathrm{j}$ ). Its condensed chromatin shows numerous small electron-transparent spaces that have various size and are regularly arranged along the whole nuclear length (Fig. 1j).

A very elongated 'connecting piece', which links the nucleus to the flagellum, is located into a deep hollow at the nucleus base $(0.45 \mu \mathrm{m}$ long) (Fig. $1 \mathrm{~h})$. It consists of a conical structure with the apex oriented towards the nucleus and the base towards the flagellum. The connecting piece is joined to the nuclear membrane through some scattered filaments. The tail, $0.3 \mu \mathrm{m}$ in diameter, contains a conventional $9 \times 2+2$ axoneme but no accessory structure (Fig. 1h, k).

\section{Discussion and conclusions}

The filiform cell shape of the spermatozoon, the cork-screw shaped acrosome, the helical nucleus surrounding an axial mitochondrion, and the $9 \times 2+2$ axonemal arrangement strictly agree with the basic plan described for the sperm of the species of the order Macrodasyida (Ferraguti and Balsamo 1995; Guidi et al. 2004).

The specific morphology of the spermatozoon of the Danish species agrees with that of the male gametes of the other two species of Lepidodasys studied so far. The acrosome structure made of two regions, a cork-screw apical one more electron-dense than the rod-like basal one, the morphology of the nucleus-mitochondrion complex, and the absence of a periaxonemal sheath (striated cylinder) are all features shared with both species of Lepidodasys studied by Guidi et al. (2004). The presence of large and irregular vesicles in the basal region of the acrosome characterizes the sperm of both the Danish species and Lepidodasys unicarenatus (Guidi et al. 2004).

On the contrary, the numerous, small spaces in the condensed chromatin and the long connecting piece joining the nucleus with the flagellum are peculiar features (autapomorphies) of the species examined.

The absence of a striated cylinder in Lepidodasys is shared only by the family Turbanellidae and by the genus Xenodasys suggesting a basal position of these taxa (Guidi et al. 2004; Marotta et al. 2005).

The similar structure of the spermatozoa of all the Lepidodasys species studied and in particular their shared autapomorphies confirm that this genus lies far from the others currently included in the family Lepidodasyidae, and strongly support the need of a revision of the taxonomic and phylogenetic position of this family (Guidi et al. 2004; Marotta et al. 2005).

Acknowledgments We would like to thank SYNTHESYS organization (The Support of European Community, Research Infrastructure activity under FP6 "Structuring the European Research Area") and the Danish Natural Science Research Council Agency (Grant 21-04-0047) that has supported this study, allowing to start a scientific collaboration between the University of Urbino (Italy) and the Zoological Museum, University of Copenhagen. We are indebted to Dr. Atsushi C. Suzuki (Japan) for his technical support with the transmission electron microscope, and for his valuable help in preparing the photographic material. We would thank Prof. Maria Balsamo for her precious advices and Dr. Loretta Guidi for constructive criticism on the first draft of the paper. Thanks are also due to anonymous referees for greatly improving the original manuscript.

\section{References}

Balsamo M, Todaro MA (2002) Gastrotricha. In: Rundle SD, Robertson AL, Schmid-Araya JM (eds) Freshwater meiofauna: biology and ecology. Backhuys, Leiden, pp 45-61

Balsamo M, Fregni E, Ferraguti M (1998) Gastrotricha. In: Adiyodi KG, Adiyodi RG (eds) Reproductive biology of the invertebrates, vol IX, Part A. Jamieson BGM (ed) Progress in male gamete ultrastructure and phylogeny. Wiley, Chichester, pp 171-192

Ferraguti M, Balsamo M (1995) Comparative spermatology of gastrotricha. In: Jamieson BGM, Ausio J, Justine JL (eds) Advances in spermatozoal phylogeny and taxonomy. Mém Mus nat Hist nat Paris 166:105-117

Guidi L, Ferraguti M, Pierboni L, Balsamo M (2003a) Spermiogenesis and spermatozoa in Acanthodasys aculeatus (Gastrotricha, Macrodasyida): an ultrastructural study. Acta Zool 84:77-85

Guidi L, Marotta R, Pierboni L, Ferraguti M, Todaro MA, Balsamo M (2003b) Comparative sperm ultrastructure of Neodasys ciritus and Musellifer delamarei, two species considered to be basal among Chaetonotida (Gastrotricha). Zoomorphology 122:135143

Guidi L, Pierboni L, Ferraguti M, Todaro MA, Balsamo M (2004) Spermatology of the genus Lepidodasys Remane, 1926 (Gastro- 
tricha, Macrodasyida): towards a revision of the family Lepidodasyidae Remane, 1927. Acta Zool 86:211-221

Hochberg R, Litvaitis MK (2000) Phylogeny of Gastrotricha: a morphology-based framework of gastrotrich relationships. Biol Bull 198:299-305

Hochberg R, Litvaitis MK (2001) Macrodasyida (Gastrotricha): a cladistic analysis of morphology. Invertebr Biol 120:124-135

Kalt MR, Tandler B (1971) A study of fixation of early amphibian embryos for electron microscopy. J Ultrastruct Res 36(5):633-645

Kristensen RM (1976) On the fine structure of Batillipes noerrevangi, 1976. 1. Tegument and moulting cycle. Zool Anz 197:129-150

Lake PS (1973) Trialdehyde fixation of crustacean tissue for electron microscopy. Crustaceana 24:244-246

Manylov OG, Vladychenskaya NS, Milyutina IA, Kedrova OS, Korokhov NP, Dvoryanchikov GA, Aleshin VV, Petrov NB (2004) Analysis of 18S rRNA gene sequences suggests significant molecular differences between Macrodasyida and Chaetonotida (Gastrotricha). Mol Phyl Evol 30:850-854

Marotta R, Guidi L, Pierboni L, Ferraguti M, Todaro MA, Balsamo M (2005) Sperm ultrastructure of Macrodasys caudatus (Gastrotri- cha: Macrodasyida) and a sperm-based phylogenetic analysis of Gastrotricha. Meiofauna Marina 14:9-21

Reynolds ES (1963) The use of lead citrate at high pH as electronopaque stain in electron microscopy. J Cell Biol 17:208-212

Rieger GE, Rieger RM (1977) Comparative fine structure study of the gastrotrich cuticle and aspects of cuticle evolution within the Aschelminthes. Z Zool Syst Evolutionsforsch 15:81-124

Ruppert EE (1978) The reproductive system of Gastrotrichs. III. Genital organs of Thaumastodermatinae subfam. n. and Diplodasyinae subfam. $n$. with discussion of reproduction in Macrodasyida. Zool Scr 7:93-114

Todaro MA, Littlewood DTJ, Balsamo M, Herniou EA, Cassanelli S, Manicardi G, Wirz A, Tongiorgi P (2003) The interrelationships of the Gastrotricha using nuclear small rRNA subunit sequence data, with an interpretation based on morphology. Zool Anz 242:145-156

Todaro MA, Telford MJ, Lockyer AE, Littlewood DTJ (2006) Interrelationships of the Gastrotricha and their place among the Metazoa inferred from 18S rRNA genes. Zool Scr 35:251-259 